\title{
A Semi-Automatic Clustering-Based Level Set Method for Segmentation of Endocardium from MSCT Images
}

\author{
Qi Su, Kwan-Yee K. Wong, George S. K. Fung
}

\begin{abstract}
Multi-slice Computed Tomography (MSCT) is an important medical imaging tool that provides dynamic three-dimensional (3D) volume data of the heart for diagnosis of various cardiac diseases. Due to the huge amount of data in MSCT, manual identification, segmentation and tracking of various parts of the heart are very labor intensive and inefficient. In this paper, we introduce a semi-automatic method for robustly segmenting the endocardium surface from cardiac MSCT images. A level set approach is adopted to define a flexible and powerful interface for capturing the complex anatomical structure of the heart. A novel speed function based on clustering the image intensities of the region of interest and the background is proposed for use with the level set method. The method introduced in this paper has the advantages of simple initialization and being capable of segmenting the blood pool with non-homogeneous intensities. Experiments on real data using the proposed speed function have been carried out with 2D, 3D and 4D implementations of the level sets respectively, and comparisons in terms of computational speed and segmentation results are presented.
\end{abstract}

\section{INTRODUCTION}

$\mathrm{T}$ o study the healthiness of a heart, various objective measurements, such as ejection fraction, ventricle blood volume, wall mass, wall motion and wall thickness over various phases of the cardiac cycle, might be required. Accurate and robust segmentation of the heart wall, especially the endocardium surface, is essential in determining the aforementioned measurements. Endocardium has a relatively complex anatomical structure, with various papillary muscles connecting between the heart wall and valves. Traditional 2D or 3D active contour methods are often not effective nor sufficient in modeling such a complicated sharp concave surface. The segmentation problem is also further complicated by the non-homogeneous distribution of contrast inside the blood pool of the heart chambers, making it virtually impossible to perform a successful segmentation using only a predefined range of intensity values. Besides, the huge amount of data in cardiac MSCT images would mean that tremendous and tedious user involvement is required in the initialization of the active contours.

In this paper, we propose a semi-automatic clustering-based level set method to robustly segment the endocardium surface from cardiac MSCT images. A novel

Qi Su and Kwan-Yee K. Wong are with the Department of Computer Science, The University of Hong Kong, Pokfulam Road, Hong Kong (phone: 852-25878454; fax: 852-25598447; e-mail: qsu@cs.hku.hk).

George S. K. Fung is with the Department of Electrical and Electronic Engineering, The University of Hong Kong, Pokfulam Road, Hong Kong (e-mail: skfung@eee.hku.hk). speed function based on clustering the image intensities of the region of interest and the background is proposed to leverage the level set method. The proposed speed function has been implemented to drive level sets of different dimensions and the segmentation results on real data are analyzed.

\section{RELATED WORK}

Digital image processing methods, such as active contours, B-spline snake and deformable templates have been exploited in the automation of medical segmentation. In recent years, level set method [1] has become a popular method due to its flexibility and capability in modeling complex structures. In [2], Paragios proposed a shape-driven level set method for segmenting the left ventricle based on a prior shape assumption. Other similar approaches using shape information with level sets include [3], [4] and [5]. In [6], Debreuve et al. introduced a level set method for segmenting the myocardial from SPECT images. An energy function of the level set equation was minimized by assuming a constant intensity approximation of the object and background regions. In [7], an intensity-based level set method was applied to a $2 \mathrm{D}+\mathrm{t}$ cardiac cine-MRI data set for analyzing the deformations of the left ventricular myocardium.

Although the above mentioned methods report successful segmentation results in their problem settings, these methods, however, cannot be directly applied to the problem considered in this paper. First of all, the endocardium has an extremely complex surface and it is virtually impossible to provide a good shape prior for its segmentation using a shape-driven level set method. Besides, the blood pool region and surrounding regions have rather non-homogeneous intensity values. This will make any segmentation method based on the assumption of constant range of intensity value fail disgracefully. Finally, our targeted application is on the segmentation of the dynamic volume dataset of cardiac MSCT, which is a $3 \mathrm{D}+\mathrm{t}$ dataset. It is important to study the performance of level set method for different dimensional domains.

In this paper, we propose to use the level set method together with a novel clustering-based speed function for extracting the endocardium surface from cardiac MSCT images. Compared with other active contour approaches, level set is good at segmenting complex surfaces as the moving interface defined in level set can merge, split or even disappear during its evolution. Instead of using a constant range of intensities, we propose to use clusters of intensity values as a better representation of the non-homogeneous 
blood pool region and surrounding anatomical structures, and derive a novel clustering-based speed function to drive the level set interface to the target surface.

The semi-automatic clustering-based level set method has been implemented and applied to segment the endocardium from the MSCT data. The segmentation can be done by applying 2D, 3D and 4D level set methods to the individual 2D CT image, 3D volume dataset of the MSCT images and 4D dynamic volume dataset of the MSCT images, respectively. Ways to initialize the level sets of different dimensions are presented, and the amounts of user intervention, computational time involved, as well as the segmentation results are analyzed.

\section{LEVEL SET BASIC THEORY}

The level set theory was explained in details by Sethian in [1]. The main idea is to track the motion of the zero set interface as the level set function evolves.

Let the moving interface be $\Gamma(t) \mathrm{t} \in[0,1]$. The level set function is set as

$$
\phi(\mathbf{x})= \pm d,
$$

where $d$ is the distance between the point $\mathbf{x}$ and $\Gamma(t)$, and the plus/minus sign is chosen depending on whether the point is outside/ inside the interface. For points on the interface, we have

$$
\phi(\mathbf{x})=0 .
$$

Applying chain rule to (1) gives

$$
\phi_{t}+F|\nabla \phi|=0
$$

where $F$ is the speed of the interface point along its normal direction. At any time the evolving interface is given by

$$
\Gamma(t)=\{\mathbf{x} \mid \phi(\mathbf{x})=0\} .
$$

The moving interface evolves under the effect of $F$. It expands when $F$ is positive, while it contracts when $F$ is negative. When $F$ is equal to zero, the interface stops and gives the segmentation result.

\section{INITIALIZATION}

Before the level set function begins to iterate, some prior information from the user is required. In our method, we need to sample the intensity values for the blood pool and the surrounding regions, and we also need to define an initial interface.

\section{A. Clustering the Intensity Values}

Our implementation allows users to sample intensities of the blood pool and the surrounding regions by drawing lines on the CT image.

The clusters for the background and for the endocardium are constructed from the intensity samples using the K-Mean algorithm [8]. The mean intensities of the clusters $K_{i o}$ and $K_{i b}$ are obtained for the foreground and the background, respectively, where $\mathrm{i}=1, \ldots, n$ and $n$ is the number of the clusters. The K-Mean method is initialized to have 32 clusters in our experiments.

\section{B. Initializing the Interface}

The main differences between the 2D, 3D and 4D level set methods are the input data and the initial interfaces.

In 2D level set, a closed curve is used for the initialization of the initial interface. To accurately segment the target, including the holes inside the target, the 2D level set method requires the initial curve passing through all the parts of the target (see Fig. 1). Suppose there are 10 sets of volume data each with 100 images, the $2 \mathrm{D}$ level set would then require 1000 different initial curves. The 2D approach therefore requires an extremely labour intensive initialization.

In 3D level set, a closed surface is used for initializing the initial interface. In defining the initial surface, the user is only

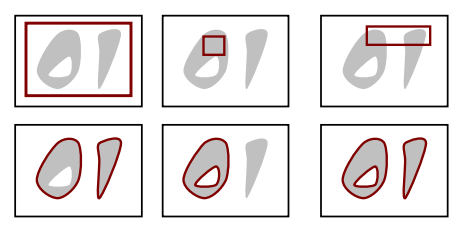

Fig. 1. In the first row, the red curves are the different initial curves. The corresponding results using $2 \mathrm{D}$ level set method are shown in the second row.

required to define a $2 \mathrm{D}$ initial curve in one image of the volume, and a $3 \mathrm{D}$ surface is then constructed by replicating and filling the 2D curve to the two neighboring images (see Fig. 2). Holes in the structure can be easily captured as the interface evolves and expands across the images within the volume. Holes segmented in one image can also be back-propagated to segment holes missed in the previous image during the evolution. Hence, as opposed to 2D level set, a simple curve enclosing the target structure can be used as the initial 2D curve. For the same data set mentioned, the 3D level set would then require only 10 different initial curves, one for each volume.

In 4D level set, a closed 4D hyper-surface is used for the initialization of the initial interface. The whole 4D dynamic

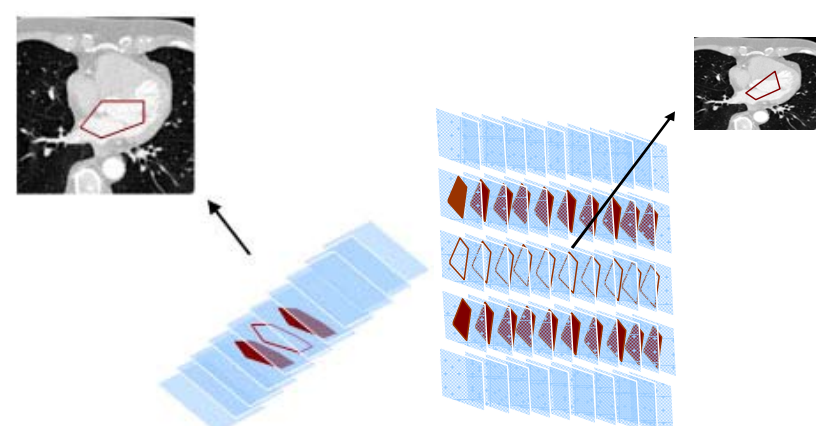

Fig. 2. Left: The initial curve defined in one image is used to construct a $3 \mathrm{D}$ surface in $3 \mathrm{D}$ level set. Right: The initial curve defined in one image is used to construct a 4D hyper-surface in 4D level set.

volume dataset can be process by one single 4D level set. In defining the initial hyper-surface, the user is only required to define a 2D initial curve in one image of the volume captured at the middle of the whole dynamic volume dataset. The initial 4D hyper-surface is then constructed and by replicating the $2 \mathrm{D}$ curve to the images of the middle volume by 
replicating and filling the $2 \mathrm{D}$ curve to the images in the neighboring volumes (see Fig. 2). The level set interface evolves in both the spatial and time domains. For the same data set mentioned, the 4D level set would only require 1 initial curve for the whole 10 volumes.

\section{Speed Function}

The speed function $F$ controls the deformation of the interface and is the most import part of the level set method. To segment the non-homogeneous structure, a clustering-based speed function is introduced in this paper. It is defined as

$$
F=F_{d}+F_{s},
$$

where $F_{\mathrm{d}}$ is related to the clusters of intensities, and $F_{s}$ controls the smoothness of the interface.

For every point $\mathbf{x}$ on the volume space, the distance from its intensity to the foreground clusters is defined as:

$$
d_{o}=\min _{i \in[0, n]}\left\|I(\mathbf{x})-K_{i o}\right\|
$$

and the distance from its intensity to the background clusters is defined as:

$$
d_{b}=\min _{i \in[0, n]}\left\|I(\mathbf{x})-K_{i b}\right\|
$$

where $K_{i o}$ and $K_{i b}$ are the mean intensities of the clusters. The term $F_{d}$ is then defined as:

$$
F_{d}=\left\{\begin{array}{l}
0, \quad \text { if } \mathbf{x} \text { is a border point and }-\varepsilon \log \left(\frac{d_{o}}{d_{b}}\right) \geq 0 . \\
-\varepsilon \log \left(\frac{d_{o}}{d_{b}}\right), \text { others. }
\end{array}\right.
$$

where $\mathcal{E}$ is a positive constant.

$F_{d}$ is designed to be positive when the intensity of the point is near to the target clusters and negative when it is close to the background clusters. Therefore, the level set interface will move inwards if it is located on the background and outwards if it is located within the target region. Eventually, the interface will stop and tightly surround the endocardium.

\section{RESULTS}

To test our method, a dynamic volume dataset of cardiac MSCT of a patient without cardiac disease was used. The dataset consists of 10 volumes which equally spaced over the phases of the cardiac cycle. Each volume has 91 CT images (i.e., with a total of $910 \mathrm{CT}$ images). A contrast enhanced with saline tracer protocol was employed. The X-ray tube was set to $120 \mathrm{kvp}$ and the reconstructed resolution was $0.4 \mathrm{~mm} \times$ $0.4 \mathrm{~mm} \times 0.6 \mathrm{~mm}$. A standard smoothing kernel was used to produce smooth mediastinum.

The segmentation results of endocardium from the cardiac MSCT images using our proposed clustering-based 2D, 3D and 4D level set methods are shown in Fig. 3. Obviously, the segmentation results obtained from the 3D and 4D level set methods are much better than that from the 2D method, with more fine details being captured. Both methods can segment the complicate endocardium surfaces with concave part and
TABLE I

PROCESSING TIME

\begin{tabular}{|r|r|r|r|}
\hline & $2 \mathrm{D}$ & $3 \mathrm{D}$ & $4 \mathrm{D}$ \\
\hline $\begin{array}{r}\text { Manual Initialization } \\
(10 \text { vol. of 91 images) }\end{array}$ & $\begin{array}{r}16 \mathrm{~s} * 910 \\
=14,560 \mathrm{~s}\end{array}$ & $\begin{array}{r}16 \mathrm{~s} * 10 \\
=160 \mathrm{~s}\end{array}$ & $16 \mathrm{~s}$ \\
\hline $\begin{array}{r}\text { Average } \\
\text { Processing time }\end{array}$ & $\begin{array}{r}0.5 \mathrm{~s} \\
\text { (per image) }\end{array}$ & $\begin{array}{r}6250 \mathrm{~s} \\
\text { (per volume) }\end{array}$ & $\begin{array}{r}60,156 \mathrm{~s} \\
\text { (whole set) }\end{array}$ \\
\hline $\begin{array}{r}\text { Total Processing time } \\
(10 \text { vol. of 91images) }\end{array}$ & $455 \mathrm{~s}$ & $62,500 \mathrm{~s}$ & $60,156 \mathrm{~s}$ \\
\hline $\begin{array}{r}\text { Manual Initialization } \\
\text { + Processing time }\end{array}$ & $15,015 \mathrm{~s}$ & $62,660 \mathrm{~s}$ & $60,172 \mathrm{~s}$ \\
\hline
\end{tabular}

holes.

Table 1 shows some statistics on the initialization and computational times for the 2D, 3D and 4D level set methods. Here we assume it takes approximately 16 seconds to draws sampling lines and initial 2D curve on one CT image. If a complicate special curve is required, which is common in the 2D level set method, it will often take longer than 16 seconds to complete the initialization. The 2D, 3D and 4D level sets require 910, 10 and 1 times of manual initialization respectively. From Table 1, it can be seen that the 2D method requires approximately 14,560 seconds to initializing $910 \mathrm{CT}$ images. Since the level set only evolves in a $2 \mathrm{D}$ domain, the processing time is negligible. Although the total time (excluding file I/Os) spent by the 2D method is much less than the 3D and 4D methods, the heavy user involvement in initialization and missing details in the final segmentation result render the 2D approach not very useful nor practical. Both the 3D and 4D methods take around $60 \mathrm{k}$ seconds to process the whole 10 sets of volume data and produce similar segmentation results. However, the 4D method only requires initializing the level set once; it is more user-friendly than the 3D method. Besides, the 4D method can, theoretically, capture more details than the 3D method as the level set can evolve in both the 3D spatial and time domains.

\section{DISSCUSSION}

From Fig. 3, it can be observed that the proposed semi-automatic clustering-based level set methods can successfully segment the endocardium surface of the left ventricle. In particular, the concave surface part, which is caused by papillary muscle, can be segmented successfully.

Our segmentation methods have integrated the advantages of level set theory and clustering algorithm which have the capability to i) segment the complex endocardium surface with extending papillary muscles, and ii) handle the non-homogenous CT number of blood pool region and the surrounding region in the cardiac MSCT images. Experimental results show that a 4D implementation of the level set can both reduce the time spent in manual initialization and improve the segmentation results.

\section{CONCLUSION}

In this paper, we present a novel clustering-based level set method for segmenting endocardium from cardiac MSCT 
images. The performance of the 2D, 3D and 4D level set methods are compared and analyzed. A novel speed function which exploits region cluster information is introduced to handle the complex endocardium surface with sharp concave structure and ensure robustness of our method. The multidimensional approach also significantly reduces the user involvement. Due to the flexibility of this approach, the algorithm is readily extendable to handle multiple cardiac anatomical structures and dynamic volumetric dataset.

\section{REFFERNCES}

[1] J. Sathian, Level set method and fast matching method, Cambridge university press. 1999.

[2] N. Paragios, "A level set approach for shape-driven segmentation and tracking of the left ventricle," IEEE Trans. Medical Imaging, 2003, pp. 773-776.
[3] M. P. Jolly, "Combining edge, region, and shape information to segment the left ventricle in cardiac MR images," in Proc. MICCAI, 2001, pp. 482-490.

[4] N. Paragios, "A variational approach for the segmentation of the left ventricle in cardiac image analysis," IJCV, vol. 50, 2002, pp. 345-362.

[5] A. Yezzi, L. Zollei, and T. Kapur, "A variational framework for joint segmentation and registration," in Proc. IEEE MMBIA, 2001, pp. 44-51.

[6] E. Debreuve, M. Barlaud, G. Aubert, and J. Darourt, "Space time segmentation using level set active contours applied to myocardial gated SPECT," IEEE Transactions on Medical Imaging, 20(7):, 2001, pp. 643-659.

[7] Y. Chenoune, E. Deléchelle, E. Petit, and J. Garot, "Validation by the HARP Method of a New Algorithm to Assess Left Ventricular Deformation on Standard Cine-MRI," Computers in Cardiology, Lyon (France), 2005.

[8] R. O. Duda, P.E. Hart and D. G. Srork, Pattern Classification, 2nded, Wiley Press, 2000

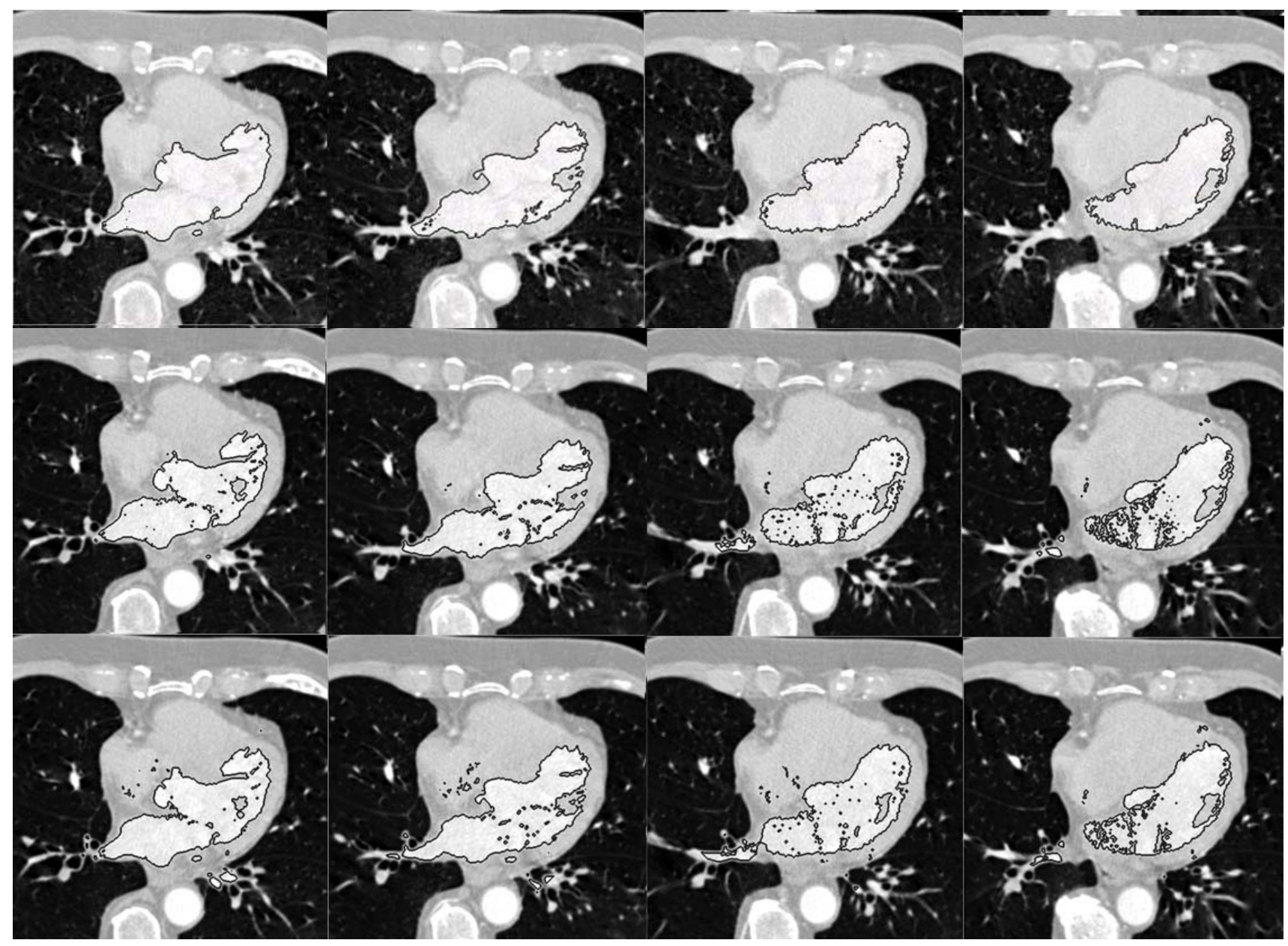

Fig. 3. The first row shows the corresponding segmentation results using the $2 \mathrm{D}$ level set method. The second row shows the corresponding segmentation results using the 3D level set method. The third row shows the corresponding segmentation results using the 4D level set method. 